\title{
Ergodic Properties of a Semi-Infinite One-Dimensional System of Statistical Mechanics
}

\author{
C. Boldrighini ${ }^{1, \star}$, A. Pellegrinotti ${ }^{2}$, E. Presutti ${ }^{3}$, Ya. G. Sinai ${ }^{4}$, \\ and M. R. Soloveichik ${ }^{5}$ \\ 1 Department of Mathematics, Rutgers University, New Brunswick, NJ 08903, USA, \\ on leave of absence from Università di Camerino, Italy \\ 2 Dipartimento di Matematica, Università di Camerino, Camerino, Italy \\ 3 Dipartimento di Matematica, Università La Sapienza, Roma, Italy \\ 4 Landau Institute for Theoretical Physics, Moscow, USSR \\ 5 Moscow State University, Moscow, USSR
}

\begin{abstract}
We consider the dynamical system $\left(\mathfrak{X}, \mu, T_{t}\right)$ where $(\mathfrak{X}, \mu)$ is the Gibbs ensemble at some fixed temperature and density for a semi-infinite onedimensional ideal gas of point particles. The first particle has mass $M$, all the other particles mass $m<M . T_{t}$ is the time evolution which describes free motion of the particles except for elastic collisions with each other and with the wall at the origin. We prove that $\left(\mathfrak{X}, \mu, T_{t}\right)$ is a $K$-flow.
\end{abstract}

\section{Introduction}

The ergodic properties of the infinite ideal gas are well established, cf. [1,2]. However there is at present no idea on how to treat interacting systems. We investigate here a somewhat intermediate system, in which interaction is confined to a finite subsystem.

More precisely, we consider an infinite system of point-like particles on the half line $R_{+}=[0, \infty)$, which interact only by elastic collisions with each other and with a "wall" placed at the origin $q=0$. We assume that the mass of the first particle, i.e. the one closest to the wall, is $M>0$, and that all the other particles have a common mass $m<M$. We shall refer to the first particle as the "heavy particle" (h.p.).

The Gibbs state for the ideal gas corresponding to any choice of the inverse temperature $\beta$ and of the particle density $\varrho$ is an equilibrium state for the system. The dynamics is most conveniently described in terms of "pulse trajectories." When two particles of mass $m$ collide we let them cross each other, keeping their velocities. The dynamics for these new objects ("pulses") is as follows: they move with constant velocity until they collide with the h.p., then they change their velocity according to the law of elastic collisions, and keep their new velocity until the next collision with the h.p. Clearly the new description is equivalent to the traditional one and it has the advantage that the interaction is reduced to collisions with the h.p.

Since the incoming flow of particles keeps the h.p. close to the wall, a pulse will interact with the h.p. maybe several times, but finally it will escape to infinity

* Partially supported by NSF Grant DMR-81-14726 
without recollisions. It would seem that we have a cluster structure: the pulses come in independent clusters, interact and go away. Therefore we would expect good ergodic properties. However since the h.p. can reach any given point, the existence of a cluster does depend on the future. In paper [3], which studies a mechanical model for a thermal bath, the difficulty is removed by the presence of a second wall at distance $L$, which is transparent for the pulses but reflects the h.p. The ergodic properties of the system are essentially due to the fact that the past is forgotten each time the h.p. reaches $L$, i.e. given its velocity at that time, the distributions of the future evolution is completely determined.

In the proof which we give below the cluster idea still plays a fundamental role. We prove that sooner or later a particular configuration of pulses arrives near the origin, such that the last moment of interaction of the h.p. with them is a "good candidate" to be a cluster time (i.e. a time after which the h.p. never collides again with any of the particles with which it collided in the past). A "good candidate" means that the probability of a recollision is very small.

It seems to us that the idea of our proof recalls what Landau and Lifshitz [4] say in the first pages of their Statistical Physics: "A subsystem of the system under consideration is a mechanical system, but certainly not a closed one. On the contrary it undergoes all kinds of interactions with other parts of the system .... Owing to their complexity it will pass sufficiently often through all its possible states ... The substantial distribution of a given subsystem is independent of the initial state of any other small part of the same system, because, after a sufficiently long time, the influence of the initial state will be completely outweighed by the influence of other, much larger parts of the system."

The plan of the paper is the following. In Sect. 2 we give definitions and state the results. We also establish the following criterion: The $K$ property is equivalent to the fact that the conditional measures, given the past history of the h.p., are equivalent when relativized to the $\sigma$-algebra generated by the behavior of the h.p. in the distant future. In Sect. 3 we prove some probabilistic results, and we show that there are cluster times in a set of full measure. In Sect. 4 we prove the equivalence required by the criterion, making use of a "copying procedure," by which we "copy" the distant future history of the h.p. in a configuration $x$ with that determined by configurations $x^{\prime}$ which can have "almost any" trajectory of the h.p. up to time $t=0$. We do this by using the existence of cluster times proved in Sect. 3 . In the Appendix we prove some probabilistic estimates.

\section{Description of the System and Formulation of the Results}

The one-particle phase space is $R_{+}^{2}=\left\{(q, v) \in R^{2} ; q>0\right\}$, where $q$ denotes the position of the particle and $v$ its velocity. $\mathfrak{X}$ denotes the space of the locally finite configurations in $R_{+}^{2}$ and $\mathfrak{M}$ the $\sigma$-algebra of the Borel sets of $\mathfrak{X}$. If $x \in \mathfrak{X}$ and $A \subseteq R_{+}^{2}$ is a measurable set we denote by $x_{A}$ the configuration $x \cap A$. $\mathfrak{M}_{A}$ denotes the sub- $\sigma$ algebra generated by $x_{A}$. By $\mathfrak{X}_{A}$ we denote the phase space of a particle system in $A$. Sometimes we shall not distinguish between $\mathfrak{X}_{A}$ and the subset $\hat{\mathfrak{X}}_{A}=\left\{x \in \mathfrak{X}: x_{R_{+}^{2} \backslash A}=\emptyset\right)$ and we shall identify the subsets of $\mathfrak{M}_{A}$ with the corresponding subsets of $\hat{\mathfrak{X}}_{A}$. A point $x \in \mathfrak{X}$ can be identified by a sequence $x=\left\{q_{k}(x), v_{k}(x)\right\}_{k=0}^{\infty}$ in which the particles are labelled in order of increasing 
position, and, for equal position, of increasing velocity. $M$ is the mass of the first particle $\left(q_{0}, v_{0}\right)$, and $m<M$ the masses of the other particles.

$\mu$ denotes the free gas Gibbs equilibrium measure corresponding to some fixed values of the particle density $\varrho$ and of the temperature $\beta$. By $\mu^{0}$ we denote the same measure for $M$ equal to $m . \mu^{0}$ is a Poisson stochastic field on $R_{+}^{2}$. By $\mu_{A}, \mu_{A}^{0}$ we shall denote the measure induced on $\mathfrak{M}_{A}, A \subseteq R_{+}^{2}$, measurable. We shall sometimes understand such measures as measures on $\mathfrak{X}_{A}$.

The dynamics can be described as follows: all particles keep their velocity until either (i) the h.p. reaches the origin and reflects its velocity, or (ii) the h.p. collides with a light one (pulse). If $V$ and $u$ are the velocities before collision of the h.p. and of the light one, respectively, we prescribe that at the collision time they have the outgoing velocities $V^{\prime}$ and $u^{\prime}$ respectively:

$$
\begin{aligned}
& V^{\prime}=\alpha V+(1-\alpha) u, \\
& u^{\prime}=-\alpha u+(1+\alpha) V,
\end{aligned}
$$

with

$$
\alpha=\frac{M-m}{M+m}
$$

We define in such a way a measure preserving flow $\left\{T_{t}\right\}_{t R}$, on an invariant set of $\mu$-measure one. We denote by $T_{t}^{0}$ the "free flow," i.e. the flow when all masses are equal to $m$, and (elastic) collisions occur only at the origin.

Our main result is the following:

Theorem 2.1. $\left(\mathfrak{X}, \mu, T_{t}\right)$ is a K-system.

To prove Theorem 2.1 we introduce the measurable partition $\zeta_{-}[5]$, generated by the random variables $q_{0}(t), t \leqq 0$, where $q_{0}(t)$ denotes the position of the h.p. at time $t$ (here and in the following, partitions should always be understood as defined $\mu$-modulo zero). By definition $T_{t} \zeta_{-} \geqq \zeta_{-}$for $t \geqq 0$,

$$
\bigvee_{t \geqq 0} T_{t} \zeta_{-}=\varepsilon
$$

$\varepsilon$ being the partition of $\mathfrak{X}$ into points. In fact with probability one all particles have nonzero velocity, so they eventually collide, in the future or in the past, with the h.p. Their velocities can therefore be reconstructed from the path of the h.p., which is measurable with respect to the partition on the left-hand side of Eq. (2.2).

To show that the system is $K$ it remains to prove that

$$
\bigwedge_{t<0} T_{t} \zeta_{-}=v
$$

where $v$ is the trivial partition. We will use a criterion given in Proposition 2.1 below, to state which we need some more notation.

If $\zeta$ is a partition we shall use the same symbol to denote the $\sigma$-algebra generated by it, and denote by $\zeta(x)$ the atom of $\zeta$ containing the point $x$. Let $\zeta^{t}$ denote the partition generated by $q_{0}\left(t^{\prime}\right), t^{\prime}>t$ and

$$
\zeta^{\infty}=\bigwedge_{t>0} \zeta^{t} .
$$


Proposition 2.1. If there is a set $E \zeta_{-}$-measurable and such that $\mu(E)=1$, and for any $x, x^{\prime} \in E$, the measures $\mu\left(\cdot \mid \zeta_{-}(x)\right)$ and $\mu\left(\cdot \mid \zeta_{-}\left(x^{\prime}\right)\right)$ are equivalent on the $\sigma$-algebra $\zeta^{\infty}$ [defined by Eq. (2.4)], then $\zeta^{\infty}$ is trivial.

Remark 2.1. Under the assumptions of Proposition $2.1\left(\mathfrak{X}, \mu, T_{t}^{-1}\right)$ is a $K$-system, and this implies that $\left(\mathfrak{X}, \mu, T_{t}\right)$ is also a $K$-system [5].

Proof of Proposition 2.1. Let $A \in \zeta^{\infty}, \mu(A)>0$. Then there is a sequence $\left(\varepsilon_{n}, B_{n}\right)$, with $\varepsilon_{n} \downarrow 0$ and $B_{n}$ cylindrical [i.e. measurable with respect to the $\sigma$-algebra generated by $q_{0}(t)$ for $t$ in a bounded interval] such that

$$
\mu\left(A \Delta B_{n}\right)<\varepsilon_{n} .
$$

(Here $A \Delta B_{n}$ denotes, as usual, the symmetric difference of the sets.) Since $B_{n}$ is cylindrical, for some negative $t_{n}$ the set $D_{n}=S_{t_{n}} B_{n}$ is $\zeta_{-}$-measurable. So for $A_{n}=S_{t_{n}} A$ we have

$$
\mu\left(A_{n} \Delta D_{n}\right)=\mu\left(A \Delta B_{n}\right) \leqq \varepsilon_{n} \Rightarrow \mu\left(A_{n}^{c} \cap D_{n}\right) \leqq \varepsilon_{n},
$$

where $A_{n}^{c}$ is the complement of $A_{n}$. From Eq. (2.6), using Chebyshev's inequality

$$
\mu\left(\left\{x \in D_{n}: \mu\left(A_{n}^{c} \mid \zeta_{-}(x)\right)>\sqrt{\varepsilon_{n}}\right\}\right) \leqq \sqrt{\varepsilon_{n}},
$$

and setting

$$
\tilde{D}_{n}=\left\{x \in D_{n}: \mu\left(A_{n}^{c} \mid \zeta_{-}(x)\right)<\sqrt{\varepsilon_{n}}\right\}=\left\{x \in D_{n}: \mu\left(A_{n} \mid \zeta_{-}(x)\right)>1-\sqrt{\varepsilon_{n}}\right\}
$$

we have, from Eq. (2.6)

$$
\mu\left(\tilde{D}_{n}\right) \geqq \mu\left(D_{n}\right)-\sqrt{\varepsilon_{n}} \geqq \mu(A)-2 \sqrt{\varepsilon_{n}} .
$$

Let $D=\lim \sup \tilde{D}_{n}$. Then by Eq. (2.7)

$$
\mu(D) \geqq \mu(A)>0 .
$$

Taking $x \in D \cap E$ there is a subsequence $n_{i}$ (depending on $x$ ) such that

$$
\lim _{i \rightarrow \infty} \mu\left(A_{n_{i}} \mid \zeta_{-}(x)\right)=1 \text {. }
$$

Since for $x^{\prime} \in E \mu\left(\cdot \mid \zeta_{-}\left(x^{\prime}\right)\right)$ is equivalent to $\mu\left(\cdot \mid \zeta_{-}(x)\right)$, we have

$$
\lim _{i \rightarrow \infty} \mu\left(A_{n_{i}} \mid \zeta_{-}\left(x^{\prime}\right)\right)=1 \quad \forall x^{\prime} \in E,
$$

where $\left\{n_{i}\right\}$ is the same sequence as in Eq. (2.8). Since $\mu(E)=1$ we have

$$
1=\int \mu\left(d x^{\prime}\right) \lim _{i \rightarrow \infty} \mu\left(A_{n_{i}} \mid \zeta_{-}\left(x^{\prime}\right)\right)=\lim _{i \rightarrow \infty} \int \mu\left(d x^{\prime}\right) \mu\left(A_{n_{i}} \mid \zeta_{-}\left(x^{\prime}\right)\right)=\mu(A) .
$$

\section{Existence of Cluster Times}

We prove in this section an intermediate result, which has interest also for itself, namely the existence of "cluster times" for a.a. $x \in \mathfrak{X}$. 
Definition 3.1. We say that $\bar{t}$ is a cluster time (c.t.) for $x \in \mathfrak{X}$ if it is a collision time for the h.p. such that the h.p. never collides for $t>\bar{t}$ with the particles with which it collided for $t \leqq \bar{t}$.

We describe the collision of the h.p. by a set of "collision parameters."

Definition 3.2. The triple $Q=(t, q, v)$, where $t$ is the time of the collision, $q$ its position and $v$ the outgoing velocity of the h.p., is the set of the collision parameters of a collision of the h.p.

It can be seen that except for a set of $\mu$-measure 0 (for which multiple collisions occur) the sequence of the collision parameters completely identifies the configuration $x \in \mathfrak{X}$.

Before proving the existence of cluster times we give three basic lemmas.

Lemma 3.1. There is a constant $c>0$ such that for $\mu$-a.a. $x \in \mathfrak{X}$,

$$
\begin{aligned}
& \lim _{t \rightarrow \pm \infty} \sup \frac{\left|v_{0}\left(T_{t} x\right)\right|}{\log ^{1 / 2}|t|}<c, \\
& \lim _{t \rightarrow \pm \infty} \sup \frac{q_{0}\left(T_{t} x\right)}{\log |t|}<c .
\end{aligned}
$$

Proof. Let $E(t)=\left\{x \in \mathfrak{X}:\left|v_{0}\left(T_{t} x\right)\right|>c \log ^{1 / 2}|t|\right\}$ with $c>\sqrt{\frac{2}{\beta m}}>\sqrt{\frac{2}{\beta M}}$. Since the distribution of $v_{0}$ is stationary with density $\sqrt{\frac{\beta M}{2 \pi}} \exp \left(-\beta M \frac{v^{2}}{2}\right)$, we have $\sum_{u=0}^{\infty} \mu(E(u))<\infty$, so that Ineq. (3.1a) holds for the integers. Now if $\left|v_{0}(x)\right|<c \log ^{1 / 2} k$ and $\left|v_{0}\left(T_{t} x\right)\right| \geqq c \log ^{1 / 2} k$ for some $t \in(0,1)$, there must be at least one particle with velocity $\bar{v}<-c \log ^{1 / 2} k$ in the region

$$
\left\{(q, v) \in R_{+}^{2}: v \in\left(-\infty,-c \log ^{1 / 2} k\right), q \in\left(q_{0}, 2 q_{0}+\left|v_{0}\right|-v\right)\right\} .
$$

If $E_{k}$ denotes the corresponding event, we have

$$
\begin{aligned}
\mu\left(E_{k}\right)= & \varrho\left(\frac{\beta M}{2 \pi}\right)^{1 / 2} \int_{0}^{\infty} d q_{0} e^{-\varrho q_{0}} \int_{-c \log ^{1 / 2} k}^{c \log ^{1 / 2} k} d v_{0} e^{-\beta M v_{0}^{2} / 2} \mu\left(E_{k} \mid q_{0}, v_{0}\right) . \\
= & \varrho\left(\frac{\beta^{2} M m}{4 \pi^{2}}\right)^{1 / 2} \int_{0}^{\infty} d q_{0} e^{-\varrho q_{0}} \\
& \cdot \int_{-c \log ^{1 / 2} k}^{c \log ^{1 / 2} k} d v_{0} e^{-\beta M v_{0}^{2} / 2} \int_{-\infty}^{-c \log ^{1 / 2} k} d v e^{-\beta m v^{2} / 2}\left(q_{0}+\left|v_{0}\right|-v\right) \leqq \frac{c_{1}}{k^{1+\delta}}
\end{aligned}
$$

for some $c_{1}>0$ and $\delta>0$. This proves Ineq. (3.1a). An analogous argument shows that Ineq. (3.1b) holds for $t$ integer, and Ineq. (3.1b) is easily deduced for continuous $t$ using Ineq. (3.1a).

Definition 3.3. For any $L>0$ and $s>t>0$ we set

$$
\begin{gathered}
C_{L}(t, s)=\left\{(q, v) \in R_{+}^{2}: q \geqq L, q+v \tau=L \text { for some } \tau \in[t, s)\right\}, \\
R_{L}(t)=R_{+}^{2} \backslash C_{L}(t, \infty) .
\end{gathered}
$$




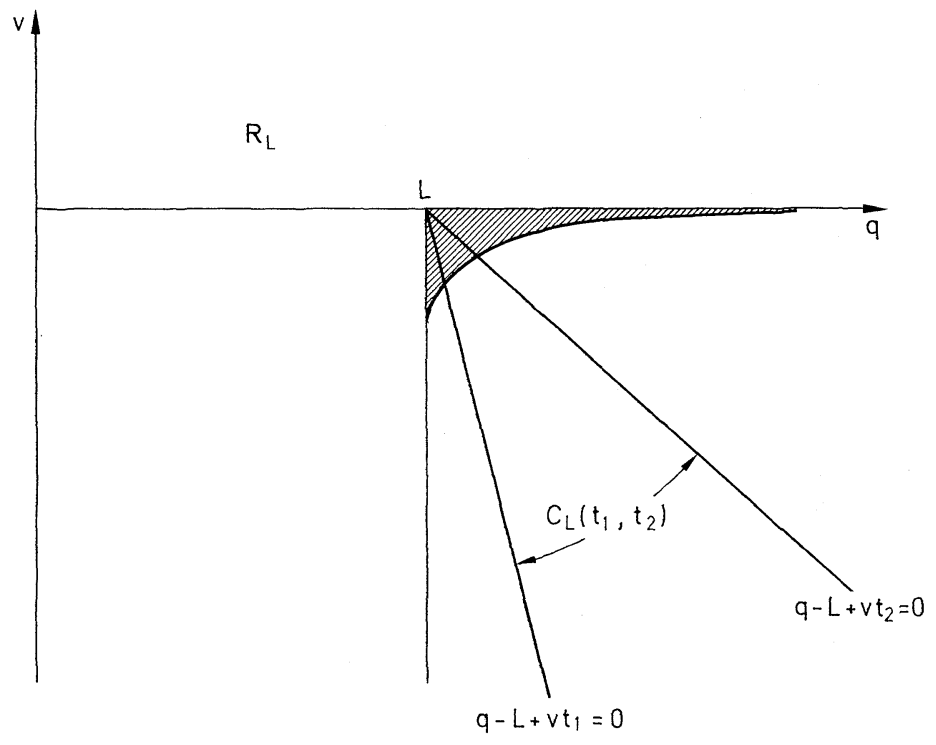

Fig. 1

$$
q-L+v t_{1}=0
$$

In what follows we shorthand $C_{L}=C_{L}(0, \infty)$ and $R_{L}=R_{L}(0, \infty)$ (see Fig. 1). $C_{L}(t, s)$ contains the particles which, under free dynamics, cross $L$ between times $t$ and $s$.

Since the h.p. is "confined" close to the wall we expect that "most" of the negative particles never collided with it. We shall now show that those which collided are a finite number, i.e. that for $\mu$-a.a. $x$ we can find an $L$ so large that all such particles are in $[0, L)$.

Lemma 3.2. Let $\mathscr{N}_{L}$ denote the subset of $\mathfrak{X}$ for which $q_{0}<L$ and all the negative particles which collided with it in the past have coordinates in $[0, L)$. Then

$$
\lim _{L \rightarrow \infty} \mu\left(\mathscr{N}_{L}\right)=1
$$

Proof. Consider the region $\Gamma_{L} \subset C_{L}$

$$
\Gamma_{L}=\left\{(q, v) \in R_{+}^{2}: q \geqq L, 0 \geqq v \geqq-\sqrt{L} e^{-((q / \sqrt{L})+1)}\right\}
$$

(see Fig. 1), and a configuration $x \in \mathfrak{U}_{L} \cap \mathcal{N}_{L}^{\prime}$, where

$$
\mathfrak{A}_{L}=\left\{x \in \mathfrak{X}: x_{\Gamma_{L}}=\emptyset\right\}
$$

is the subset for which $\Gamma_{L}$ is empty, and

$$
\mathscr{N}_{L}^{\prime}=\left\{x \in \mathfrak{X}: q_{0}\left(T_{t} x\right)<\sqrt{L} \log _{+}|t|, t<0\right\} .
$$

Then the h.p. in $x$ did not collide with the particles of $x_{C_{L}}$ in the past. In fact, suppose that such collisions took place, and that $t_{0}$ is the last collision time. Clearly $t_{0}<0$ and for $t_{0}<t \leqq 0$ we have

$$
T_{t} x=T_{t} x_{R_{L}} \cup T_{t}^{0} x_{C_{L}} .
$$

So for $t \in\left(t_{0}, 0\right]$ the particles of $x_{C_{L}}$ are in the region $C_{L}(-t, \infty) \backslash \Gamma_{L}(-t)$, where

$$
\Gamma_{L}(\tau)=\left\{(q, v) \in R_{+}^{2}: q+v \tau \in \Gamma_{L}\right\}
$$


is the preimage of $\Gamma_{L}$. But a simple computation shows that

$$
\inf \left\{q:(q, v) \in C_{L}(\tau, \infty) \cap \Gamma_{L}(\tau)\right\} \geqq \max \left(L, \sqrt{L} \log _{+} \tau\right) .
$$

So there is no such collision, or $t_{0}=-\infty$. Therefore $\mathscr{N}_{L} \supset \mathscr{N}_{L}^{\prime} \cap \mathfrak{A}_{L}$. By Lemma 3.1 $\lim _{L \rightarrow \infty} \mu\left(\mathscr{N}_{L}^{\prime}\right)=1$. Moreover, since the Lebesgue measure of $\Gamma_{L}$ is

$$
\operatorname{meas}\left(\Gamma_{L}\right)=L e^{-(\sqrt{L}+1)} \underset{L \rightarrow \infty}{\longrightarrow} 0,
$$

we have

$$
\lim _{L \rightarrow \infty} \mu\left(\mathfrak{A}_{L}\right)=1
$$

which proves the result.

Remark 3.1. Clearly $\mathscr{N}_{L} \in \zeta_{-}$. Moreover if $\zeta_{L}$ denotes the partition generated by $x_{R_{L}}$, then its restriction to $\left.\mathscr{N}_{L} \zeta_{L}\right|_{\mathscr{N}_{L}}$ is a refinement of $\left.\zeta_{-}\right|_{\mathscr{N}_{L}}:\left.\zeta_{L}\right|_{\mathscr{N}_{L}} \geqq\left.\zeta_{-}\right|_{\mathscr{N}_{L}}$.

Remark 3.2. It is easy to see that $\mathcal{N}_{L}^{\prime} \cap \mathfrak{A}_{L}=\mathscr{E}_{L} \cap \mathfrak{A}_{L}$, where $\mathscr{E}_{L} \in \mathfrak{M}_{R_{L}}\left(=\zeta_{L}\right)$ is given by

$$
\mathscr{E}_{L}=\left\{x \in \mathfrak{X}: q_{0}\left(T_{t} x_{R_{L}}\right)<\sqrt{L} \log _{+}|t|, t \leqq 0\right\},
$$

because there are no collisions with $x_{C_{L}}$, and as a simple consequence of the proof above we get

$$
\lim _{L \rightarrow \infty} \mu\left(\mathscr{E}_{L}\right)=1
$$

The following simple lemma shows that the infimum of the positions of the positive particles goes away faster than any power $t^{\gamma}, \gamma<1 / 2$.

Lemma 3.3. For $a>0, \gamma \in(0,1 / 2)$, and $t_{0}>0$, consider the set

$$
V_{a, t_{0}}=\left\{(q, v) \in[a, \infty) \times[0, \infty): \min _{t>t_{0}} q+v t-a-t^{\gamma} \leqq 0\right\} .
$$

Then

$$
\lim _{t_{0} \rightarrow \infty} \mu\left(\left\{x: x_{V_{a, t_{0}}}=\emptyset\right\}\right)=1,
$$

and the limit is uniform in $a \in[0, \infty)$.

Proof. $V_{a, t_{0}}$ is the region between the half-line $[a, \infty)$ on the $q$ axis and the curve

$$
v(q)=\left\{\begin{array}{lll}
\frac{t_{0}^{\gamma}-(q-a)}{t_{0}} & \text { for } & q-a \leqq(1-\gamma) t_{0}^{\gamma} \\
\gamma\left(\frac{q-a}{1-\gamma}\right)^{1-(1 / \gamma)} & \text { for } & q-a \geqq(1-\gamma) t_{0}^{\gamma} .
\end{array}\right.
$$

It is easily seen that for $(1 / \gamma)-1>1$ the Lebesgue measure of $V_{a, t_{0}}$ goes to 0 uniformly in $a$ as $t_{0} \rightarrow \infty$, which proves the lemma.

We can now state the main result of this section. 
Theorem 3.1. There is a set $E^{\prime}, \mu\left(E^{\prime}\right)=1$, such that for $x \in E^{\prime}$ there is an infinite number of positive cluster times.

Proof. The theorem is proved by proving the following two statements.

Statement 3.1. If $L>0$ is large enough there is a set $\hat{\mathfrak{X}}_{L}^{(0)} \subset \mathfrak{X}, \mu\left(\hat{\mathfrak{X}}_{L}^{(0)}\right)>0$ such that for $x \in \hat{\mathfrak{X}}_{L}^{(0)}$ there is a c.t. with parameter $Q=(\bar{t}, \bar{q}, \bar{v})$ such that $\bar{t} \in(0,1)$, $\bar{q} \in(2 L / 3,(\varepsilon+2 / 3) L), \varepsilon=L^{-1 / 6}, \bar{v}<0$.

Statement 3.2. There is a set $\hat{\mathfrak{X}}_{L} \subset \bigcup_{\tau \geqq 0} T_{-\tau} \hat{\mathfrak{X}}_{L}^{(0)}$ such that

$$
\lim _{L \rightarrow \infty} \mu\left(\hat{\mathfrak{X}}_{L}\right)=1 \text {. }
$$

In fact, take an increasing sequence $L_{n}$ such that $\mu\left(\hat{\mathfrak{X}}_{L n}\right)>1-2^{-n}$. Then by the Borel-Cantelli Theorem the set

$$
E^{\prime}=\lim _{n \rightarrow \infty} \inf \hat{\mathfrak{X}}_{L n}
$$

has the required properties.

Proof of Statement 3.1. We construct $\hat{\mathfrak{X}}_{L}^{(0)}$, by giving separate conditions on $x_{R_{L}}$ and $x_{C_{L}}$. To make the text legible we give some reference or commentary for each condition.

A. Conditions on $x_{R_{L}}$

a1) $x \in \mathscr{E}_{L / 2}:$ [see Eq. (3.10)];

a2) $x_{R_{L}} \cap \Gamma_{L / 2}=\emptyset:[$ see Eq. (3.7)];

a3) $x \in \mathscr{B}_{L}^{\prime}: \mathscr{B}_{L}^{\prime} \in \mathfrak{M}_{R_{L}}$ is defined in the appendix [Eq. (A.1)], and is a set satisfying some condition of "uniform distribution" for the particles in $[0, L)$;

a4) $x \in \mathscr{B}_{L}^{\prime \prime}: \mathscr{B}_{L}^{\prime \prime} \in \mathfrak{M}_{R_{L}}$ is defined in the appendix (Eq. A.2) and is the set for which the absolute value of the velocities in $[0, L)$ does not exceed $c \log ^{1 / 2} L$ for some constant $c>0$;

a5) $x \in \mathscr{V}_{L}$, where

$$
\mathscr{V}_{L}=\left\{x \in \mathfrak{X}: \min _{\substack{(q, v) \in x \\ q>2 L / 3, v>0}} q+v t>2 L / 3+t^{2 / 5} \text { for } t>r_{L}\right\}
$$

for

$$
r_{L}=(2 L / 3)^{2 / 5}
$$

We denote the set on which conditions a1)-a5) are satisfied by $\hat{\mathscr{E}}_{L}^{(0)}$. Clearly $\hat{\mathscr{E}}_{L}^{(0)} \in \mathfrak{M}_{R_{L}}$, and by Lemmas 3.3 and A.1, Eqs. (3.8) and (3.11) it follows that

$$
\lim _{L \rightarrow \infty} \mu\left(\hat{\mathscr{E}}_{L}^{(0)}\right)=1
$$

B. Conditions on $X_{C_{L}\left(0, r_{L}\right)}$. The region $C_{L}\left(0, r_{L}\right)$ (given by Definition 3.3) contains the negative particles which cross $L$ before time $r_{L}$. We want them to be arranged in a very special way so that they can produce a c.t. The construction (as well as the "copying" construction of next section) is made possible by the fact that, as it follows from the collision equations (2.1), if we let $u \rightarrow-\infty$ for fixed $V, u^{\prime} \rightarrow+\infty$ (and $\left.V^{v^{\prime}} \rightarrow-\infty\right)$, i.e. a very fast incoming particle reflects from the h.p. almost as from a 
"wall", i.e. preserving a high energy. This clearly depends on the fact that $\alpha>0$, i.e. $M>m$. For $M \leqq m$ our arguments do not apply, although, presumably, the results are still valid.

We shall describe the particles in $C_{L}\left(0, r_{L}\right)$ as well as the effect which they produce.

b1) A particle $(q, v),-v(1-\alpha) \in\left(e^{c L}, e^{c L}+1\right)$ and $q+v t_{0}=L$, for some

$$
c>\bar{c}=\varrho \log (1 / \alpha) t_{0} \in(0,1 / 2) .
$$

It follows from Proposition A.1 of the Appendix that if $x \in \mathscr{B}_{L}^{\prime} \cap \mathscr{B}_{L}^{\prime \prime}$, then for $L$ large the h.p. in $\hat{x}_{L}=x_{R_{L}} \cup(q, v)$ after colliding with $(q, v)$ reflects from the wall and never after reverses its velocity. Moreover, since $|v|$ is large, the motion of the h.p. becomes "almost deterministic" (on scale $L$ ), which implies (Corollary A.1) that there is a time $\hat{t}$, depending only on $(q, v)$ such that $\left|\hat{t}-t_{0}\right|<2 L e^{-\lambda L}, 0<\lambda$ $<\min (\bar{c}, c-\bar{c})$, and at time $\hat{t}$ the h.p. is in the interval $(2 L / 3,(\varepsilon+2 / 3) L)$ with velocity $V \in\left(V_{m}, V_{M}\right), V_{m}=(1-\alpha)|v| \alpha^{2 L(1+\varepsilon) / 3}, V_{M}=(1-\alpha)|v| \alpha^{2 L(1-\varepsilon) / 3}, \varepsilon=L^{-1 / 6}$. Furthermore all the particles which collide with it in $[0, L)$ get a velocity larger than $w=e^{\lambda L}$.

b2) A second particle $\left(q_{1}, v_{1}\right)$ such that

$$
q_{1}+\hat{t} v_{1} \in(2 L / 3,(\varepsilon+2 / 3) L) \text { and }-v_{1}(1-\alpha) \in\left((1+\alpha) V_{M}, 2 V_{M}\right)
$$

In the configuration $x_{R_{L}} \cup(q, v) \cup\left(q_{1}, v_{1}\right),\left(q_{1}, v_{1}\right)$ it will collide at some place

$$
\bar{q} \in(2 L / 3,(\varepsilon+2 / 3) L)
$$

and at some time $\bar{t},|\hat{t}-\bar{t}|<\varepsilon L \frac{1-\alpha}{1+\alpha} \frac{1}{V_{M}}$. Hence $\bar{t}<1$ for $L$ large. Moreover the outgoing velocity $V_{1}$ of the h.p. is such that $-V_{1} \in\left(V_{M}, 2 V_{M}\right)$.

b3) A sequence $\left(q_{i}, v_{i}\right), i=2,3, \ldots, N$, which collides with the h.p. after time $\bar{t}$ in such a way that after hitting the wall the first time it is kept in $[0, L / 2)$ with velocity less in modulus than $\tilde{V}=1+\frac{2 \alpha}{1-\alpha} V_{M}$ until time $r_{L}-1$. The sequence can be realized in the following way. Since $|\hat{t}-\bar{t}|=0\left(L \varepsilon / V_{M}\right)$ and $V_{1} \in\left(-2 V_{M},-V_{M}\right)$, there is an interval $\left(\hat{t}_{1}, \hat{t}_{2}\right)$ depending only on $(q, v)$ such that for $t \in\left(\hat{t}_{1}, \hat{t}_{2}\right)$ the h.p. is in $[0, L / 2)$ and $\hat{t}_{2}-\hat{t}_{1}<\frac{1}{4} \frac{L}{V_{M}}$. So if we take $\left(q_{2}, v_{2}\right)$ such that

$$
v_{2} \in\left(-\frac{\alpha}{1-\alpha} 2 V_{M}-1,-\frac{\alpha}{1-\alpha} 2 V_{M}\right)
$$

and $q_{2}+\tau v_{2}=L / 2$ for some $\tau \in\left(\hat{t}_{1}, \hat{t}_{2}\right)$, it collides in $[0, L / 2)$, and the outgoing velocity of the h.p. will be negative. To choose the other ones take the first integer $\bar{n}$ such that $\alpha^{\tilde{n}}<1 / 2$ and $\delta$ so small that $2 \alpha^{n}<1-\delta / \tilde{V}$. Then choose $v_{k} \in(-\tilde{V},-\tilde{V}+\delta)$ and $q_{k}$ such that for the times $t_{k}=\frac{1}{\left|v_{k}\right|}\left(q_{k}-\frac{L}{2}\right)$ the inequalities $t_{k}-t_{k-1}<\frac{L}{2 \widetilde{V} \tilde{n}}$ hold for $k=3,4, \ldots, 1$. It is easy to see that the h.p. by colliding with such particles and with the negative particles of $x_{R_{L}}$ which are left at time $\bar{t}$ (right of $2 L / 3$ ) never gets a velocity larger (in absolute value) than $\tilde{V}$. If it starts off at the wall with such velocity, then because $t_{k}-t_{k-1}$ is so small, it must undergo at least $\bar{n}$ collisions 
before reaching $L / 2$, which are enough to make it go back. So it cannot cross $L / 2$ before colliding with the last particle of the sequence.

b4) A sequence of at least $\tilde{N}>\log \tilde{V} / \log (1 / \alpha)$ particles with velocity between -1 and -2 which at time $r_{L}-1$ are in $(L / 2, L)$.

We denote the set for which $x_{C_{L}\left(0, r_{L}\right)}$ satisfies conditions b1)-b4) by $\mathscr{F}_{L}^{(0)}$. Clearly $\mathscr{F}_{L}^{(0)} \in \mathfrak{M}_{C_{L}\left(0, r_{L}\right)}$ and

$$
\mu^{0}\left(\mathscr{F}_{L}^{(0)}\right)>0
$$

Remark 3.3. For $x \in \mathscr{F}_{L}^{(0)} x_{C_{L}\left(0, r_{L}\right)} \cap \Gamma_{L / 2}=\emptyset$ for $L$ large, since $x_{C_{L}\left(0, r_{L}\right)}$ does not contain any particle $(q, v)$ with $|v|<1$.

Remark 3.4. By b3) at time $r_{L}-1$ the h.p. is in $[0, L / 2)$ with velocity less than $\tilde{V}$ in absolute value, and by condition b4) there are enough slow negative particles in $(L / 2, L)$ to make it go back with velocity less than 2 in absolute value. So we conclude that i) at time $r_{L}$ it is still in $[0, L)$ and ii) it cannot get out of $L$ with velocity larger than 2 unless it collides with particles which are not in $x_{R_{L}} \cup x_{C_{L}\left(0, r_{L}\right)}$

\section{Conditions on $x_{C_{L}\left(r_{L}, \infty\right)}$}

c1) $x_{C_{L}\left(r_{L}, \infty\right)} \cap \Gamma_{L / 2}=\emptyset$. This condition, together with conditions a2) and Remark 3.2 ensures that $x \in \mathscr{N}_{L / 2}$. By the construction above the h.p. between times $\bar{t}$ and $r_{L}$ does not collide with particles with which it collided before $\bar{t}$.

So $\bar{t}$ is a c.t. unless it is spoiled by recollisions at times $t>r_{L}$. Such recollisions can be i) with the particles of $x_{R_{L}}$ which at time $\bar{t}$ were to the right of $\bar{q}$, and ii) with other particles of $x_{R_{L}}$ and with the two incoming particles of points b1) and b2). By condition a5) the particles of group i) are at least at distance $2 L / 3+t^{2 / 5}$ from the origin. (The particles of group ii) go away with very high velocity, so that by time $r_{L}$ they will be farther away than that, and we don't have to take account of them.) Our next condition is stated in the following result.

Lemma 3.4. There is a set $\mathscr{U}_{L} \in \mathfrak{M}_{C_{L}\left(r_{L}, \infty\right)}, \lim \mu\left(\mathscr{U}_{L}\right)=1$, such that if $x \in \mathscr{U}_{L}$ and satisfies conditions a1)-a5), b1)-b4) above, then $q_{0}\left(T_{t+r_{L}} x\right)<L+L / 4+\log ^{2} t$ for $t \geqq r_{L}$.

Proof. First note that in order to get a bound for $q_{0}\left(T_{t+r_{L}} x\right)$ we cannot use equilibrium estimates, since at time $r_{L}$ the situation in $[0, L)$ is certainly not typical. We use the fact that for the h.p. to cross a point $a>L$ before time $r_{L}+T$ there must be a time $t \in\left[r_{L}, r_{L}+T\right)$ at which it starts off at $q=0$ with a velocity $V$ so large that $M V>\pi_{a}(t, t+a / V)$, where

$$
\pi_{a}(t, t+s)=m \sum_{(q, v) \in C_{a}(t, t+s)}|v| \quad t, s>0
$$

is the absolute value of the total momentum of the negative particles which cross $a$ between times $t$ and $t+s$.

We shall prove that we can take

$$
\mathscr{U}_{L}=\left\{x: x_{C_{L}\left(r_{L}, \infty\right)} \in T_{-r_{L}}^{0} \mathscr{U}_{L}^{\left(k_{0}\right)}\right\} \quad k_{0}=[2 L / 9],
$$

where $\mathscr{U}_{L}^{\left(k_{0}\right)}$ is defined by Eq. (A.11) of the Appendix. In fact we know that at time $r_{L}$ the h.p. is still in $[0, L)$. Suppose that at some time $t>r_{L}$ it starts off the wall and 
reaches $L+k$ before time $t_{k}=e^{\sqrt{k}}, k=1,2, \ldots$, without inverting its velocity. If $k$ is so large that

$$
v_{k}=(2 / \beta m)\left(\sqrt{k}+\log \left(1 / k^{2}\right)\right)>2 \text { and } x_{C_{L}\left(r_{L}, \infty\right)} \in T_{-r_{L}}^{0} \mathscr{R}_{k}^{\prime \prime}(L)\left(\supset T_{-r_{L}}^{0} \mathscr{U}_{L}^{\left(k_{0}\right)}\right)
$$

[see Eq. (A.9)] this cannot happen unless the h.p. collided in the time interval $\left(r_{L}, t\right]$ with some particle of $x_{C_{L}\left(r_{L}, \infty\right)}$ with velocity larger than $v_{k}$ in modulus. Indeed, otherwise the h.p. would start with a velocity less than $2<v_{k}$, and it would be driven back by the particles crossing $L+k$ (see Proposition A.2). So if $T_{r_{L}}^{0} x_{C_{L}\left(r_{L}, \infty\right)} \in \mathscr{R}_{k}^{\prime \prime}(L)$ it cannot cross $L+k$ before time $t_{k}$ unless $T_{r_{L}}^{0} x_{C_{L}\left(r_{L}, \infty\right)} \in \overline{\mathscr{R}_{k}^{\prime}(L)}$. Now if $L$ is large $v_{k_{0}}>2$ for $k_{0}=[2 L / 9]$ and if $T_{r_{L}}^{0} x_{C_{L}\left(r_{L}, \infty\right)} \in \mathscr{U}_{L}^{\left(k_{0}\right)}, k \geqq k_{0}$ we find

$$
\sup _{0 \leqq t \leqq \tau} q\left(T_{t+r_{L}} x\right) \leqq L+1+k_{0}+\log ^{2} \tau \leqq 5 L / 4+\log ^{2} \tau \text {. }
$$

The fact that $\lim _{L \rightarrow \infty} \mu\left(\mathscr{U}_{L}\right)=1$ is an easy consequence of Eq. (A.16) of the Appendix.

We can now state our second condition:

c2) $x \in \mathscr{U}_{L}$. Note that, since $5 L / 4+\log ^{2} \tau<2 L / 3+\left(\tau+r_{L}\right)^{2 / 5}$, if conditions a1)-a5), b1)-b4), and c1)-c2) are satisfied there is actually a c.t.

We denote by $\hat{\mathfrak{U}}_{L}^{(0)}$ the set for which conditions c1)-c2) hold. Clearly $\hat{\mathfrak{U}}_{L}^{(0)} \in \mathfrak{M}_{C_{L}\left(r_{L}, \infty\right)}$ and, by Eq. (3.8), Lemma 3.4, and Remark A.1 of the Appendix we have

$$
\lim _{L \rightarrow \infty} \mu\left(\hat{\mathfrak{U}}_{L}^{(0)}\right)=\lim _{L \rightarrow \infty} \mu^{0}\left(\hat{\mathfrak{U}}_{L}^{(0)}\right)=1 .
$$

The proof of Statement 3.1 is accomplished by setting

$$
\hat{\mathfrak{X}}_{L}^{(0)}=\hat{\mathscr{E}}_{L}^{(0)} \cap \mathscr{F}_{L}^{(0)} \cap \hat{\mathfrak{P}}_{L}^{(0)},
$$

since we have

$$
\mu\left(\hat{\mathfrak{X}}_{L}^{(0)}\right)=\mu\left(\hat{\mathscr{E}}_{L}^{(0)} \mid \mathscr{F}_{L}^{(0)} \cap \hat{\mathfrak{A}}_{L}^{(0)}\right) \mu^{0}\left(\mathscr{F}_{L}^{(0)} \cap \hat{\mathfrak{A}}_{L}^{(0)}\right)=\mu\left(\hat{\mathscr{E}}_{L}^{(0)}\right) \mu^{0}\left(\mathscr{F}_{L}^{(0)}\right) \mu^{0}\left(\hat{\mathfrak{A}}_{L}^{(0)}\right)>0
$$

Proof of Statement 3.2. Statement 3.2 would follow from Statement 3.1 if we had an ergodic theorem. Since we don't, we give an explicit construction of the set $\hat{\mathfrak{X}}_{L}$. The main point consists in the observation that for $T_{\tau} x \in \hat{\mathfrak{X}}_{L}^{(0)}$ for some $\tau>0$, it is enough, in most cases, that $T_{\tau} x_{R_{L}(\tau)} \in \hat{\mathscr{E}}_{L}^{(0)}$ [see Definition (3.3)] and

$$
T_{\tau}^{0} x_{C_{L}(\tau, \infty)} \in \mathscr{F}_{L}^{(0)} \cap \hat{\mathfrak{U}}_{L}^{(0)},
$$

i.e. we can assume the particles outside $R_{L}(\tau)$ to move freely. This is not surprising for small $\tau$, but it is true for $\tau$ arbitrarily large, because, even if the h.p. can go by time $\tau$ much farther than $L$, it is nevertheless unlikely that it collides with the particles of $x_{C_{L}(\tau, \infty)}$.

Consider the sets [see Eqs. (3.3), (3.4)]

$$
\begin{aligned}
\mathscr{E}_{L}^{(\tau)}=\left\{x \in \mathfrak{X}: q_{0}\left(T_{t+\tau} x_{R_{L}(\tau)}\right)\left(\sqrt{L} \log _{+}(t), t \leqq 0\right)\right\}, \\
\mathfrak{U}_{L}^{(\tau)}=T_{-\tau}^{0} \mathfrak{U}_{L}=\left\{x \in \mathfrak{X}: x_{\Gamma_{L}(\tau)}=\emptyset\right\} .
\end{aligned}
$$

Following the proof of Lemma 3.2 it is easily shown that for $x \in \tilde{N}(\tau)=\mathscr{E}_{L}^{(\tau)} \cap \mathfrak{U}_{L}^{(\tau)}$ the h.p. does not interact with the particles of $x_{C_{L}(\tau, \infty)}$ for $-\infty<t<\tau$. Again the main point in the proof is the inequality

$$
\min \left\{q:(q, v) \in C_{L}(\tau, \infty) \cap \Gamma_{L}(\tau)\right\} \geqq \max \left(L, \sqrt{L} \log _{+} \tau\right)
$$


which, by the way, implies $\left(q_{0}, v_{0}\right) \in R_{L}(\tau)$ for $x \in \tilde{\mathscr{N}}_{L}(\tau)$ and that $\left(T_{\tau}^{0} x\right)_{C_{L} \backslash \Gamma_{L}}$ contains no particles of $x_{R_{L}(\tau)}$. Moreover we have

$$
\tilde{\mathscr{N}}_{L}^{(\tau)}=\left\{x \in \mathfrak{X}: q_{0}\left(T_{t+\tau} x\right)<\sqrt{L} \log _{+}|t|, t \leqq 0\right\} \quad \mathfrak{U}_{L}^{(\tau)}=T_{-\tau} \mathcal{N}_{L}^{\prime} \cap \mathfrak{U}_{L}^{(\tau)},
$$

which, since the Lebesgue measure of $\Gamma_{L}(\tau)$ does not depend on $\tau$, by Lemma 3.2, Eq. (3.8) and the invariance of $\mu$ under $T_{\tau}$, gives

$$
\lim _{L \rightarrow \infty} \mu\left(\tilde{\mathscr{N}}_{L}(\tau)\right)=1
$$

Set now

$$
\widetilde{\mathscr{E}}_{L}^{(\tau)}=\left\{x \in \mathfrak{X}: T_{\tau} x_{R_{L}(\tau)} \in \hat{\mathscr{E}}_{L}^{(0)} \cap \mathfrak{A}_{L / 2}\right\} \cap \mathscr{E}_{L / 2}^{(\tau)},
$$

where $\hat{\mathscr{E}}_{L}^{(0)}$ is defined by conditions a1)-a5). Making use of the fact that for $x \in \widetilde{\mathscr{N}}_{L / 2}(\tau)$ the particles of $x_{C_{L / 2}(\tau, \infty)}$, moving by free dynamics, cannot get into $\Gamma_{L}$ at time $\tau$, and of Eqs. (3.9), (3.16), and (3.25) we get

$$
\mu\left(\widetilde{\mathscr{E}}_{L}^{(\tau)}\right)>\mu\left(\widetilde{\mathscr{E}}_{L}^{(\tau)} \cap \mathcal{N}_{L / 2}(\tau)\right)=\mu\left(\mathscr{N}_{L / 2}(\tau) \cap T_{-\tau}\left(\hat{\mathscr{E}}_{L}^{(0)} \cap \mathfrak{U}_{L / 2}\right)\right) \underset{L \rightarrow \infty}{\longrightarrow} 1,
$$

uniformly in $\tau \in[0, \infty)$. By the same argument for the set

$$
\hat{\mathscr{E}}_{L}^{(\tau)}=\widetilde{\mathscr{E}}_{L}^{(\tau)} \cap \mathscr{E}_{L / 2} \cap\left\{x \in \mathfrak{X}: x_{R_{L}(\tau)} \cap \Gamma_{L / 2}=\emptyset\right\},
$$

we get

$$
\lim _{L \rightarrow \infty} \inf _{\tau \geqq 0} \mu\left(\hat{\mathscr{E}}_{L}^{(\tau)}\right)=1
$$

Consider now the set

$$
\hat{\mathfrak{X}}_{L}^{(\tau)}=\hat{\mathscr{E}}_{L}^{(\tau)} \cap \mathscr{F}_{L}^{(\tau)} \cap \hat{\mathfrak{A}}_{L}^{(\tau)},
$$

where $\mathscr{F}_{L}^{(\tau)}=T_{-\tau}^{0} \mathscr{F}_{L}^{(0)}, \hat{\mathfrak{U}}_{L}^{(\tau)}=T_{-\tau}^{0} \hat{\mathfrak{P}}_{L}^{(0)}$, and $\hat{\mathfrak{U}}_{L}^{(0)}$ is given by conditions c1)-c2)above. We prove that for all $x \in \hat{\mathfrak{X}}_{L}^{(\tau)}$ there is a cluster time by proving that

$$
T_{\tau} \hat{\mathfrak{X}}_{L}^{(\tau)} \subset \hat{\mathfrak{X}}_{L}^{(0)} \text {. }
$$

Inclusion (3.29) follows from the two relations $\hat{\mathfrak{X}}_{L}^{(\tau)} \subset \widetilde{\mathscr{E}}_{L}^{(\tau)}$ and $\hat{\mathfrak{X}}_{L}^{(\tau)} \subset \widetilde{\mathscr{N}}_{L / 2}(\tau)$. The first one is obvious. For the second one observe that for $x \in \hat{\mathfrak{X}}_{L}^{(\tau)}$, the regions $\Gamma_{L / 2}(\tau)$ $\cap C_{L}\left(\tau+r_{L}, \infty\right)$ and $\Gamma_{L / 2}(\tau) \cap C_{L}\left(\tau, \tau+r_{L}\right)$ are empty because $x \in \hat{\mathfrak{U}}_{L}^{(\tau)}$ and $x \in \mathscr{F}_{L}^{(\tau)}($ see Remark 3.3). So since $x \in \hat{\mathscr{E}}_{L}^{(\tau)} \subset \mathscr{E}_{L}^{(\tau)}$, it follows $x \in \tilde{\mathscr{N}}_{L / 2}(\tau)$. The proof of Statement 3.2 will be accomplished by the proof that for the set

$$
\hat{\mathfrak{X}}_{L}=\bigcup_{k=0}^{\infty} \hat{\mathfrak{X}}_{L}^{(k)}
$$

we have

$$
\lim _{L \rightarrow \infty} \mu\left(\hat{\mathfrak{X}}_{L}\right)=1 .
$$

For this we need Proposition 3.1 below. Before that we state a remark.

Remark 3.5. $\hat{\mathfrak{X}}_{L}^{(\tau)} \subset \mathscr{E}_{L / 2} \cap \mathfrak{A}_{L / 2} \subset \mathscr{N}_{L / 2}$. This follows by the definition (3.26) and by the fact that

$$
\Gamma_{L / 2} \cap C_{L}\left(\tau+r_{L}, \infty\right) \subset \Gamma_{L / 2}(\tau) \cap C_{L}\left(\tau+r_{L}, \infty\right)
$$


Proposition 3.1. For any $\varepsilon>0$ there is $L_{\varepsilon}$ so large that

$$
\mu\left(\bigcup_{k=0}^{\infty}\left(\hat{\mathscr{E}}_{L}^{(k)} \cap \mathscr{F}_{L}^{(k)}\right)\right)>1-\varepsilon .
$$

Proof. The sets $\mathscr{F}_{L}^{\left(k_{j}\right)}$, where $k_{j}=j([r]+1), j=0,1,2, \ldots$ are independent with respect to the measure $\mu^{0}$ and

$$
\mu^{0}\left(\mathscr{F}_{L}^{\left(k_{j}\right)}\right)=\mu^{0}\left(\mathscr{F}_{L}^{(0)}\right)>0 .
$$

So, by the Borel-Cantelli lemma

$$
\mu^{0}\left(\bigcup_{k=0}^{\infty} \mathscr{F}_{L}^{\left(k_{j}\right)}\right)=1
$$

Therefore there is some $k_{0}$ so large that $\mu^{0}\left(\bigcup_{k=0}^{k_{0}} \mathscr{F}_{L}^{(k)}\right)>1-\varepsilon / 2$. Consider the sets

$$
F_{L}^{\left(k_{0}\right)}=\mathscr{F}_{L}^{\left(k_{0}\right)}, \quad F_{L}^{\left(k_{0}-j\right)}=\left.\mathscr{F}_{L}^{\left(k_{0}-j\right)}\right|_{i=0} ^{j-1} \mathscr{F}_{L}^{\left(k_{0}-i\right)}, \quad j=1,2, \ldots, k_{0} .
$$

Clearly $F_{L}^{(k)} \in \mathfrak{M}_{C_{L}(k, \infty)}, k=0,1, \ldots, k_{0}$, and

$$
\bigcup_{k=0}^{k_{0}}\left(\hat{\mathscr{E}}_{L}^{(k)} \cap \mathscr{F}_{L}^{(k)}\right) \supset\left(\hat{\mathscr{E}}_{L}^{(k)} \cap F_{L}^{(k)}\right)
$$

Since the sets $F_{L}^{(k)}$ are disjoint, we have

$$
\begin{aligned}
& \mu\left(\bigcup_{k=0}^{k_{0}}\left(\hat{\mathscr{E}}_{L}^{(k)} \cap \mathscr{F}_{L}^{(k)}\right)\right) \geqq \sum_{k=0}^{k_{0}} \mu\left(\hat{\mathscr{E}}_{L}^{(k)} \cap F_{L}^{(k)}\right) \\
& \quad=\sum_{k=0}^{k_{0}} \mu\left(\hat{\mathscr{E}}_{L}^{(k)}\right) \mu^{0}\left(F_{L}^{(k)}\right) \geqq(1-\varepsilon / 2) \mu^{0}\left(\bigcup_{k=0}^{k_{0}} F_{L}^{(k)}\right)>1-\varepsilon,
\end{aligned}
$$

where we used the fact that $\mu\left(F_{L}^{(k)} \mid \hat{\mathscr{E}}_{L}^{(k)}\right)=\mu^{0}\left(F_{L}^{(k)}\right)$ if $\hat{\mathscr{E}}_{L}^{(k)} C\left\{x: q_{0}(x)<L\right\}$ and we took $L$ so large that $\mu\left(\hat{\mathscr{E}}_{L}^{(k)}\right)>1-\varepsilon / 2$.

Proof of Eq. (3.31). By Proposition 3.1 for $L$ large and suitable $k_{0}$,

$$
\mu\left(\bigcup_{k=0}^{k_{0}}\left(\hat{\mathscr{E}}_{L}^{(k)} \cap \mathscr{F}_{L}^{(k)}\right)\right)>1-\varepsilon / 2 .
$$

Consider the sets

$$
\begin{aligned}
\mathscr{G}_{L}^{(0)} & =\hat{\mathscr{E}}_{L}^{(0)} \cap \mathscr{F}_{L}^{(0)}, \\
\mathscr{G}_{L}^{(1)} & =\left(\hat{\mathscr{E}}_{L}^{(1)} \cap \mathscr{F}_{L}^{(1)}\right) \backslash\left(\hat{\mathscr{E}}_{L}^{(0)} \cap \mathscr{F}_{L}^{(0)}\right), \ldots, \\
\mathscr{G}_{L}^{\left(k_{0}\right)} & =\left(\hat{\mathscr{E}}_{L}^{\left(k_{0}\right)} \cap \mathscr{F}_{L}^{\left(k_{0}\right)}\right) \backslash \bigcup_{j=0}^{k_{0}-1}\left(\hat{\mathscr{E}}_{L}^{(j)} \cap \mathscr{F}_{L}^{(j)}\right) .
\end{aligned}
$$

Clearly the sets $\mathscr{G}_{L}^{(k)} \in \mathfrak{M}_{R_{L}(k)}$ are disjoint and

$$
\bigcup_{k=0}^{k_{0}}\left(\hat{\mathscr{E}}_{L}^{(k)} \cap \mathscr{F}_{L}^{(k)} \cap \hat{\mathfrak{U}}_{L}^{(k)}\right) \supset \bigcup_{k=0}^{k_{0}}\left(\mathscr{G}_{L}^{(k)} \cap \hat{\mathfrak{U}}_{L}^{(k)}\right)
$$


so that

$$
\begin{aligned}
\mu\left(\hat{\mathfrak{X}}_{L}\right) & \geqq \sum_{k=0}^{k_{0}} \mu\left(\mathscr{G}_{L}^{(k)} \cap \hat{\mathfrak{U}}_{L}^{(k)}\right)=\sum_{k=0}^{k_{0}} \mu\left(\mathscr{G}_{L}^{(k)}\right) \mu^{(0)}\left(\hat{\mathfrak{V}}_{L}^{(k)}\right) \\
& \geqq(1-\varepsilon / 2) \mu\left(\bigcup_{k=0}^{k_{0}} \mathscr{G}_{L}^{(k)}\right)>1-\varepsilon,
\end{aligned}
$$

which proves Eq. (3.31).

\section{Proof of Theorem 2.1 by a Copying Procedure}

Consider the sets

$$
\mathfrak{X}_{L_{n}}=\left\{x: \mu\left(\hat{\mathfrak{X}}_{L_{n}} \mid \zeta_{-}(x)\right)>1-2^{-n / 2}\right\},
$$

where $\hat{\mathfrak{X}}_{L}$ is given by Eq. (3.30), the partition $\zeta_{L}$ is defined in Remark 3.1, and the sequence $L_{n}, n=1,2, \ldots, n$ is the one used in defining the set $E^{\prime}$ [Eq. (3.13)]. [Our notation for the conditional probability suggests its interpretation as a measure on the partition atom $\zeta_{-}(x)$. We recall that we often use the same notation for the partition and the $\sigma$-algebra generated by it, which for $\zeta_{L}$ is $\mathfrak{M}_{R_{L}}$.

Consider the set

$$
E=\lim _{n \rightarrow \infty} \inf _{L_{n}} .
$$

Clearly $E \in \zeta_{\text {- }}$ and by a Borel-Cantelli argument we see that $\mu(E)=1$.

In the rest of this section we shall prove that the set $E$ defined by Eq. (4.2) satisfies the assumptions of Proposition 2.1, which by Remark 2.1 accomplishes the proof of Theorem 2.1. Namely we shall prove the following result.

Proposition 4.1. For any $x^{(1)}, x^{(2)} \in$ E the measures $\mu\left(\cdot \mid \zeta_{-}\left(x^{(1)}\right)\right)$ and $\mu\left(\cdot \mid \zeta_{-}\left(x^{(2)}\right)\right)$ are equivalent on $\zeta^{\infty}$.

Proof. The proof is based on the following lemma which we shall prove later. [We recall that by $\zeta(x)$ we denote the atom of the partition $\zeta$ which contains $x$.]

Lemma 4.1. Suppose that for any $L>0$ and $x, x^{\prime} \in \hat{\mathfrak{X}}_{L}$ the condition:

$$
\mu\left(A \cap \hat{\mathfrak{X}}_{L} \mid \zeta_{L / 2}(x)\right)>0
$$

implies $\mu\left(A \cap \hat{\mathfrak{X}}_{L} \mid \zeta_{L / 2}\left(x^{\prime}\right)\right)>0$ for $A \in \zeta^{\infty}$. Then Proposition 4.1 holds.

In fact, suppose that $x^{(1)}, x^{(2)} \in E$ and that $\mu\left(A \mid \zeta_{-}\left(x^{(1)}\right)\right)>0$. We can take $n_{0}$ so large that for $n \geqq n_{0}$ i) $x^{(1)}, x^{(2)} \in \mathfrak{X}_{L_{n}}$, and ii) $\mu\left(A \cap \hat{\mathfrak{X}}_{L_{n}} \mid \zeta-\left(x^{(1)}\right)\right)>0$. Since $\hat{\mathfrak{X}}_{L} \subset \mathscr{N}_{L / 2}$ and by Remark 3.1, $\left.\zeta_{L / 2}\right|_{\mathscr{N}_{L / 2}} \geqq\left.\zeta_{-}\right|_{\mathscr{N}_{L / 2}}$, we have

$$
\mu\left(A \cap \hat{\mathfrak{X}}_{L_{n}} \mid \zeta_{-}\left(x^{(1)}\right)\right)=\int_{\zeta-\left(x^{(1)}\right) \cap \hat{\mathfrak{X}}_{L_{n}}} \mu\left(A \cap \hat{\mathfrak{X}}_{L_{n}} \mid \zeta_{L / 2}(x)\right) \mu\left(d x \mid \zeta_{-}\left(x^{(1)}\right)\right)>0 .
$$

Similarly we have

$$
\mu\left(A \cap \hat{\mathfrak{X}}_{L_{n}} \mid \zeta_{-}\left(x^{(2)}\right)\right)=\int_{\zeta-\left(x^{(2)}\right) \cap \hat{\mathfrak{X}}_{L_{n}}} \mu\left(A \cap \hat{\mathfrak{X}}_{L_{n}} \mid \zeta_{L / 2}\left(x^{\prime}\right)\right) \mu\left(d x^{\prime} \mid \zeta_{-}\left(x^{(2)}\right)\right) .
$$

Since $x^{(2)} \in \mathfrak{X}_{L_{n}} \mu\left(\hat{\mathfrak{X}}_{L_{n}} \mid \zeta_{-}\left(x^{(2)}\right)>1-2^{-n / 2}>0\right.$, so that we get $\mu\left(A \cap \hat{\mathfrak{X}}_{L_{n}} \mid \zeta_{-}\left(x^{(2)}\right)\right)>0$, and the conclusion. 
Proof of Proposition 4.1. The conditional measure $\mu\left(\cdot \mid \zeta_{L / 2}(x)\right)$ for $x \in \hat{\mathfrak{X}}_{L}$ does not depend on $x$ and is a Poisson measure, i.e. $\mu\left(\cdot \mid \zeta_{L / 2}(x)\right)=\mu_{C_{L / 2}}^{0}$. In what follows we set for brevity $Y=\mathfrak{X}_{C_{L / 2}}$ and $m=\mu_{C_{L / 2}}^{0}$. Let now $A \in \zeta^{\infty}$ and $x \in \hat{\mathfrak{X}}_{L}$ be such that $\mu\left(A \cap \hat{\mathfrak{X}}_{L}^{(k)} \mid \zeta_{L / 2}(x)\right)>0$. This implies that for some $k \mu\left(A \cap \hat{\mathfrak{X}}_{L}^{(k)} \mid \zeta_{L / 2}(x)\right)>0$, and therefore the set $\Phi^{(k)}=\left\{y: x_{R_{L / 2}} \cup y \in A \cap \hat{\mathfrak{X}}_{L}^{(k)}\right\}$ has positive $m$-measure $m\left(\Phi^{(k)}\right)>0$. From now on $k$ is fixed. By the construction of $\hat{\mathfrak{x}}_{L}^{(k)}$ in Sect. 3, we can divide $y \in \Phi^{(k)}$ into two parts: $y=w \cup z$, where $w$ denotes the particles which collide with the h.p. up to the cluster time $t_{c}=k+\bar{t}(\bar{t} \in(0,1))$, which corresponds to the collision of condition b2), and $z$ denotes the particles which collide after that. We introduce the spaces $W=\bigcup_{j=0}^{\infty}\left(C_{L / 2}\right)^{j} \equiv \bigcup_{j=0}^{\infty} W^{(j)}$ and $Z=Y$, so that $(w, z) \in W \times Z$ for all $y \in \Phi^{(k)}$. Let $P^{(k)}(d w, d z)$ denote the joint distribution of $w$ and $z$ induced by the measure $\chi_{\Phi^{(k)}}(y) m(d y)$ ( $\chi$ denotes as usual the indicator function). By the properties of the Poisson measure it is easy to see that $P^{(k)}(d w, d z) \ll \operatorname{mes}(d w)$ $\times m(d z)$, where mes is the standard measure: the restriction to $W^{(j)}$, mes ${ }^{(j)}$, is the measure induced on $W^{(j)}$ by the Lebesgue measure in $R^{2 j}$, and $\operatorname{mes}\left(W^{(0)}\right)=1$. So

$$
P^{(k)}(d w, d z)=f(w, z) \operatorname{mes}(d w) m(d z)
$$

and $f$ can be chosen in such a way that $\Phi^{(k)}=\operatorname{supp} f$. A point $w \in W^{(j)}$ can be identified with a point of $R^{2 j}$ and, for mes-a.a. points $w$ such that $(w, z) \in \operatorname{supp} f$, there is a neighborhood $\mathscr{U}(w) \subset R^{2 j}$ such that the collision parameters $Q_{c}$ (see Definition 3.2) corresponding to the cluster time $t_{c}$, are $C^{\infty}$ functions of $w$ in $\mathscr{U}(w)$. Clearly there is at least one such neighborhood $\mathscr{U} \subset R^{2 j}$ for some $j$, such that $\int_{\mathcal{U} \times z} f(w, z) \operatorname{mes}(d w) \operatorname{mes}(d z)>0$, and so the set

$$
\Phi=\left\{(w, z) \in \Phi^{(k)}: w \in \mathscr{U}\right\}
$$

has positive $m$-measure, $m(\Phi)>0$. (For brevity, we do not distinguish here between sets of $R^{2 j}$ and their images under symmetrization in $W^{(j)}$.) We can suppose $\mathscr{U}$ so small that there is a time $\tau$ which for all $w \in \mathscr{U}$ is larger than $t_{c}$, smaller than the subsequent time of collision with the wall and moreover such that the h.p. at time $\tau$ lies in the interval $(L / 3, L / 2)$.

In the configuration $x_{R_{L / 2}} \cup w \cup z$ for $(w, z) \in \Phi$ the position and velocity of the h.p. at time $\tau, \lambda=\left(q_{0}(\tau), v_{0}(\tau)\right)$, are functions of $w$ only, $\lambda=\phi(w)$, since the particles of $z$, by the construction of Sect. 3, collide with the h.p. only after it reflects from the wall, and the map $\phi: \mathscr{U} \rightarrow R_{+}^{2}$ is $C^{\infty}$ and of full rank, since $\operatorname{det}\left(\frac{\partial \phi(w)}{\partial\left(q_{1}, v_{1}\right)}\right) \neq 0$, where $\left(q_{1}, v_{1}\right)$ is the last particle of $w$ to collide with the h.p., as described in condition b2). Therefore the joint distribution $v$ of $\lambda$ and $z$ induced by the restriction of $P(d w, d z)$ to $\mathscr{U} \times Z$ is absolutely continuous with respect to mes $(d \lambda) \times m(d z)$ [where mes $(d \lambda)$ denotes the Lebesgue measure]:

$$
v(d \lambda, d z)=g(\lambda, z) \operatorname{mes}(d \lambda) m(d z),
$$

and we can assume that $g(\lambda, z)=0$ unless there is a $w$ such that $\phi(w)=\lambda$ and $(w, z) \in \Phi$. As a consequence, the marginal distribution of $\lambda, \varrho(d \lambda)=v(d \lambda, z)$, is absolutely continuous with respect to mes $(d \lambda)$. Let $\lambda^{*}=\left(q^{*}, v^{*}\right)$ be a density point for it. This implies that for any neighborhood $\mathscr{U}^{*}$ of $\lambda^{*}$ we have

$$
\int \chi_{\mathscr{U}^{*}}(\lambda) g(\lambda, z) \operatorname{mes}(d \lambda) m(d z)>0,
$$


i.e. the set $\Phi^{*} \subset \Phi$,

$$
\Phi^{*}=\left\{(\lambda, z): \lambda \in \mathscr{U}^{*}, g(\lambda, z)>0\right\}
$$

has positive $v$-measure. Moreover if $Z_{\mathscr{U}^{*}}=\left\{z: v\left(\Phi^{*}\right)>0\right\}$, by Fubini's theorem we have that $m\left(Z_{\mathscr{U}^{*}}\right)>0$, and we can assume that $\Phi^{*} \subset \mathscr{U}^{*} \times Z_{\mathscr{U}^{*}}$. In what follows we shall only consider a neighborhood of the type

$$
\mathscr{U}_{\delta}^{*}=\left\{\lambda=(q, v):\left|q-q^{*}\right|<\delta,\left|v-v^{*}\right|<\delta\right\},
$$

where $\lambda^{*}=\left(q^{*}, v^{*}\right)$ is a fixed density point, and we set $Z_{\delta}=Z_{\mathscr{U}_{\dot{\sigma}}^{*}}$

Copying Procedure. We now take another point $x^{\prime} \in \hat{\mathfrak{X}}_{L}$, and we are going to construct a neighborhood $\hat{\mathscr{U}} \subset W$ such that the trajectory of the h.p. for the configurations $x_{R_{L / 2}} \cup w \cup z$ for $(w, z) \in \Phi^{*}$ will be "copied," at large times, by the trajectories of the h.p. of the configurations $x_{R_{L / 2}}^{\prime} \cup \hat{w} \cup z, \hat{w} \in \hat{W}$. To do that we first construct a configuration $\hat{\bar{w}}$, depending on $x_{R_{L / 2}}^{\prime}$, made of the following particles:

1) A particle $\left(\hat{q}_{0}, \hat{v}_{0}\right)$ with $\hat{q}_{0}>L / 2$ and $\hat{v}_{0}<0$ such that in the configuration $x_{R_{L / 2}}^{\prime} \cup\left(\hat{q}_{0}, \hat{v}_{0}\right)$ the h.p. collides with $\left(\hat{q}_{0}, \hat{v}_{0}\right)$ at time $\hat{t}_{0}<k r_{L}$, and, after bouncing off the wall i) it collides with all the particles which at time $t$ were in the interval $[0, L / 2)$, before they reach the point $3 L / 5$, ii) impresses them a positive velocity $>1$, and iii) reaches $3 L / 5$ at a time $\hat{t}_{1}=\tau-\left(1 / V^{*}\right)\left(3 L / 5-q^{*}\right)$;

2) A second particle $\left(\hat{q}_{1}, \hat{v}_{1}\right)$ with $\hat{q}_{1}>3 L / 5$ and $\hat{q}_{1}+\hat{t}_{1} \hat{v}_{1}=3 L / 5$ such that in the collision at $3 L / 5$ the h.p. is left with velocity 0 . We can assume $\left|\hat{v}_{0}\right|$ so large that the particle $\left(\hat{q}_{1}, \hat{v}_{1}\right)$ bounces off with a velocity $\hat{v}_{1}^{\prime}>1$;

3) A third particle $\left(\hat{q}_{2}, \hat{v}_{2}\right), \hat{q}_{2}=3 L / 5-t^{*} v_{2}, v_{2}=v^{*} /(1-\alpha)$, which clearly collides with the h.p. at time $t^{*}$ and gives to it a velocity $v^{*}$ such that at time $\tau$ it is in $q^{*}$.

Clearly it is possible to determine $\hat{\bar{w}}$ for a particular $x^{\prime} \in \hat{\mathfrak{X}}_{L}$ in such a way that all the conditions above hold. Consider now a neighborhood $\hat{\mathscr{U}}$ of $\hat{\bar{w}}$, and the map $\hat{\phi}(\hat{w})=\hat{\lambda}=(\hat{q}, \hat{v})$, which gives position and velocity of the h.p. in $T_{\tau}\left(x_{R_{L / 2}}^{\prime} \cup \hat{w}\right)$ as a function of $\hat{w}$. Clearly we can choose $\hat{\bar{w}}$ and $\hat{\mathscr{U}}$ in such a way that the $\operatorname{map} \hat{\phi}: \hat{\mathscr{U}} \rightarrow R_{+}^{2}$ is $C^{\infty}$ and of full rank, and moreover such that $\phi_{\lambda}(\hat{\mathscr{U}})=\mathscr{U}_{\delta}^{*}$ (possibly by taking a small $\delta$ ).

Remark 4.1. The particles of $\hat{w} \in \hat{\mathscr{U}}$ are located in a region of $R_{+}^{2}$ different from that of the particles of $z \in Z_{\delta}$. This is because if $(q, v) \in z \in Z_{\delta}, q+\tau v>3 L / 5$, whereas a particle $\left(q^{\prime}, v^{\prime}\right) \in \hat{w}$ has to be in the region $q^{\prime}+\tau v<L / 2$.

The following observations show that the above construction achieves our aim of "copying."

i) For $L$ large $\hat{w} \cap \Gamma_{L / 2}=\emptyset$ for $\hat{w} \in \hat{\mathscr{U}}$, so that for $z \in Z_{\delta}$ and $x^{\prime} \in \hat{\mathfrak{X}}_{L}$ we have $x_{R_{L / 2}}^{\prime} \cup \hat{w} \cup z \subset \mathscr{N}_{L / 2}$, and there are no particles of the past right of $L / 2$.

ii) If $(w, z) \in \Phi$ and $\hat{w}$ is such that $\lambda=\phi(w)=\hat{\lambda}=\varphi(\hat{w})$, then the h.p. in $x_{R_{L / 2}}^{\prime} \cup \hat{w} \cup z$ will never catch up, after time $\tau$, the particles with which it collided previously. In fact at time $\tau$ it has the same position and velocity as in $x_{R_{L / 2}} \cup w \cup z$, and until time $k+r_{L}-1$ it has the same story. After $k+r_{L}$ the bound (3.20) clearly holds, because $z \in Z_{\delta}$, and, as in Sect. 3, we see that the h.p. can never reach the particles of its past because $x^{\prime} \in \hat{\mathfrak{X}}_{L}$ and the particles of $\hat{w}$ have got a high positive velocity. So the trajectories of $x_{R_{L / 2}}^{\prime} \cup \hat{w} \cup z$ and $x_{R_{L / 2}} \cup w \cup z$ coincide for $t \geqq \tau$, and since $x_{R_{L / 2}} \cup w \cup z \in A$, so does $x_{R_{L / 2}}^{\prime} \cup \hat{w} \cup z$. 
By observation ii) Proposition 4.1 will be proved if we prove the following statement.

Statement 4.1. The set $\hat{\Phi}=\left\{(\hat{w}, z) \in \hat{\mathscr{U}} \times Z_{\delta}:(\hat{\phi}(\hat{w}), z) \in \Phi^{*}\right\}$ has positive measure $m(\hat{\Phi})>0$.

Proof. The result will follow if we prove that $R(\hat{\Phi})>0$, where $R(d w, d z)=\operatorname{mes}(d w)$ - $m(d z)$. Now we know that for $z \in Z_{\delta} \quad v\left(\Phi^{*} \mid z\right)>0$ which implies $\operatorname{mes}\left(\left\{\lambda \in \mathscr{U}_{\delta}^{*}: g(\lambda, z)>0\right\}\right)>0$. Since we know that the map $\hat{\phi}: \hat{\mathscr{U}} \rightarrow \mathscr{U}_{\delta}^{*}$ is $C_{\infty}$ and of full rank, and $\hat{\phi}(\hat{\mathscr{U}})=\mathscr{U}_{\delta}^{*}$, we conclude that $\operatorname{mes}(\{\hat{w}: g(\hat{\phi}(\hat{w}), z)>0\})>0$, which proves the result.

\section{Appendix}

We prove the "almost deterministic" behavior for very fast particles in Proposition A.1, after proving a lemma.

\section{Lemma A.1. Set}

$$
\begin{gathered}
\kappa(L)=\left[L^{1 / 5}\right], \ell=L / \kappa(L), s=\ell^{3 / 5}, I_{k}=[(k-1) \ell, k \ell], \quad k=1,2, \ldots, \kappa(L), \\
\mathscr{B}_{L}^{(k)}=\left\{x \in \mathfrak{X}:|| x_{I_{k} \times R}|-\varrho \ell|<s\right\},
\end{gathered}
$$

and

$$
\begin{gathered}
\mathscr{B}_{L}^{\prime}=\bigcap_{k=1}^{\kappa(L)} \mathscr{B}_{L}^{(k)}, \\
\mathscr{B}_{L}^{\prime \prime}=\left\{x \in \mathfrak{X}: \max _{(q, v) \in x_{[0, L) \times R^{1}}}|v| \leqq c \log ^{1 / 2} L\right\} .
\end{gathered}
$$

Then there is a constant $c>0$ such that $\lim _{L \rightarrow \infty} \mu\left(\mathscr{B}_{L}\right)=1$, where $\mathscr{B}_{L}=\mathscr{B}_{L}^{\prime} \cap \mathscr{B}_{L}^{\prime \prime}$.

Proof. ( $|x|$ denotes here the cardinality of a configuration $x$.) Since the measure induced by $\mu$ on the particle positions is the usual Poisson measure on $R_{+}$with intensity $\varrho$ we have

$$
\mu\left(\mathscr{B}_{L}^{\prime}\right)=\prod_{k=1}^{\kappa(L)} \mu\left(\mathscr{B}_{L}^{(k)}\right)=\left(\mu\left(\mathscr{B}_{L}^{(1)}\right)\right)^{\kappa(L)} .
$$

Moreover, using the Chebyshev inequality for the exponential function $\exp \left\{\lambda\left|x_{I_{1} \times R^{1}}\right|\right\}$ and minimizing in $\lambda$, we get (a bar denotes the complementary set)

$$
\mu \overline{\left(\mathscr{B}_{L}^{(1)}\right)} \leqq \exp \{-\varrho \ell G(s /(\varrho \ell))\},
$$

where $G=(1+x) \log (1+x)-x$ is a $C^{\infty}$ function in $R_{+}$such that $G(0)=G^{\prime}(0)=0$ and $G^{\prime \prime}(0)=1$. So we find

$$
\left(\mu\left(\mathscr{B}_{L}^{(1)}\right)\right)^{\kappa(L)} \geqq(1-\exp \{-\varrho \ell G(s /(\varrho \ell))\})^{\kappa(L)} \underset{L \rightarrow \infty}{\longrightarrow} 1,
$$

and $\lim _{L \rightarrow \infty} \mu\left(\mathscr{B}_{L}^{\prime}\right)=1$. Moreover by the properties of the measure $\mu$ it is easy to see that

$$
\mu\left(\mathscr{B}_{L}^{\prime \prime}\right) \geqq \exp \left\{-c_{0} L \int_{|v|>\log 1 / 2 L} e^{-\beta m v^{2} / 2} d v\right\} \geqq \exp \left\{-c_{0}^{\prime} L /\left(L^{\left(c^{\prime}\right)^{2}}\right)\right\},
$$

and the last expression goes to 1 as $L \rightarrow \infty$ if $c^{\prime}=c \sqrt{\beta m}>1$. 
Proposition A.1. Let $x \in \mathscr{B}_{L}$ and set $\hat{x}=x_{R_{L}} \cup(q, v)$ with $-v(1-\alpha) \in\left(e^{c L}, e^{c L+1}\right)$, $c>\bar{c}=\varrho \log (1 / \alpha)$, and $q+v t_{0}=L$ for some $t_{0} \in(0,1 / 2)$. Then for L large enough the evolution of $\hat{x}$ is such that

i) after colliding with $(q, v)$ the h.p. hits the wall at a time $t_{1}$ and for $t>t_{1}$ never inverts its velocity;

ii) $(q, v)$ and all the particles which collide after time $t_{1}$ and are in $[0, L)$ get a positive velocity larger than $e^{\lambda L}, 0<\lambda<\min (c, c-\bar{c})$;

iii) after time $t_{1}$ the h.p. crosses the points $k \ell+c \log ^{1 / 2} L$ with a velocity smaller than $V_{M}(k)$, at a time larger than $t_{m}(k)$, and crosses the points $k \ell-c \log ^{1 / 2} k$ with a velocity larger than $V_{m}(k)$ and at times not exceeding $t_{M}(k), k=1,2, \ldots, k(L)$, where the quantities

$$
\begin{aligned}
& V_{M}(k)=(1-\alpha)|v| \alpha^{k \ell \varrho(1-\sigma)}\left(1+\varepsilon_{L}\right), \\
& V_{m}(k)=(1-\alpha)|v| \alpha^{k \ell \varrho(1-\sigma)}\left(1-\varepsilon_{L}\right)
\end{aligned}
$$

and for $k>1$

$$
\begin{aligned}
& t_{m}(k)=t_{0}+\frac{L}{|v|}+\frac{\ell}{(1-\alpha)|v|}\left(\frac{1}{\alpha}\right)^{(k-1) \varrho \ell(1-\sigma)}\left(1-\varepsilon_{L}\right), \\
& t_{M}(k)=t_{0}+\frac{L}{|v|}+\frac{\ell}{(1-\alpha)|v|}\left(\frac{1}{\alpha}\right)^{k \varrho \ell(1+\sigma)}\left(1+\varepsilon_{L}\right)
\end{aligned}
$$

do not depend on $x \in \mathscr{B}_{L}, \varepsilon_{L}=e^{-\lambda L^{4 / 5}}, \lambda \in(0, c-\bar{c})$, and $\sigma=s / \varrho \ell$.

Proof. The absolute value of the velocity of the h.p. until it collides with $(q, v)$ and of all the other particles which collide with the h.p. after time $t_{1}$ in $[0, L)$ is less than $V_{0}=c \log ^{1 / 2} L$. The only possible exception is the $(q, v)$ particle itself, which may collide (for $\alpha>1 / 2$ ) twice with the h.p. However the analysis below is not changed by adding a collision with a positive particle, and we will neglect this fact. By the collision laws $(2.1)$ we see that after colliding with $(q, v)$ the h.p. has a velocity $-V_{1}$ with

$$
V_{1}=(1-\alpha)|v|\left(1+\varepsilon_{1}\right), \quad\left|\varepsilon_{1}\right|<e^{-\lambda L}, \quad \lambda \in(0, c),
$$

and after time $t_{1}$ and before crossing $L$ its velocity is always larger than

$$
\alpha^{n}(1-\alpha)|v|\left(1+e^{-\lambda L}\right)-V_{0}>w=e^{\lambda L}, \quad \lambda \in(0, c-\bar{c})
$$

[where $n$ is the number of particles in $[0, L)]$, which proves assertions i) and ii).

When it collides with $(q, v)$ the h.p. can be at most a distance $V_{0}$ from its position at time 0 and therefore we have

$$
0 \leqq t_{1}-t_{0}-\frac{L}{|v|}<\frac{\ell+V_{0}}{|v|(1-\alpha)} .
$$

By Ineq. (A.6) it follows that the particles which collide with the h.p. after time $t_{1}$ in $[0, L)$ are at most at a distance $V_{0}$ from their position at time 0 . So when the h.p. crosses $k \ell+V_{0}$ it has already collided with all the particles which were at time 0 in $[0, k \ell)$ and its velocity cannot exceed the value

$$
\alpha^{k \varrho \ell(1-\sigma)} V_{1}+V_{0} \leqq V_{M}(k) \text {. }
$$


Similarly when it crosses $k \ell-V_{0}$ it has collided at most with all the particles which were in $[0, k \ell)$ and its velocity will be larger than

$$
\alpha^{k \varrho \ell(1+\sigma)} V_{1}-V_{0}>V_{m}(k) .
$$

Therefore the time at which it crosses $k \ell+V_{0}$ is larger than

$t_{1}+\frac{\ell+V_{0}}{V_{1}}+\frac{\ell}{V_{M}(1)}+\ldots+\frac{\ell}{V_{M}(k-1)}=t_{1}+\frac{V_{0}}{V_{1}}+\frac{\ell}{(1-\alpha)|v|} \frac{\beta^{k}-1}{\beta_{-}-1} \frac{1}{1+\varepsilon_{L}}>t_{m}(k)$,

where $\beta_{-}=(1 / \alpha)^{\varrho \ell(1-\sigma)}$, and we suppose $k>1$ and make use of Ineq. (A.7).

In a similar way one sees that the time at which it crosses $k \ell-V_{0}$ cannot exceed

$$
t_{1}+\frac{\ell-V_{0}}{V_{m}(1)}+\frac{\ell}{V_{m}(2)}+\ldots+\frac{\ell}{V_{m}(k-1)} \leqq t_{M}(k)
$$

and assertion iii) is proved.

Corollary A.1. Let $\hat{x}$ be as in Proposition A.1. Then for L large enough there is a $\hat{t}>t_{1}$ depending only on $(q, v)$ such that $\left|\hat{t}-t_{0}\right|<2 L e^{-\lambda L}$ and at time $\hat{t}$ the h.p. is in the interval $(2 L / 3,(\varepsilon+2 / 3) L)$ with a velocity $V \in\left(V_{m}, V_{M}\right)$ for $V_{m}=(1-\alpha)|v| \alpha^{(2 L / 3)(1+\varepsilon) \varrho(1-\sigma)}, V_{M}=(1-\alpha)|v| \alpha^{(2 L / 3)(1-\varepsilon) \varrho(1-\sigma)}, \varepsilon=L^{-1 / 6}$.

Proof. Set $k_{0}=\left[\frac{2}{3} \frac{L}{\ell}\right]+2$. Since $\sigma k_{0} \leqq 2 \sigma+\frac{2}{3} \frac{L}{\ell} \sigma=O\left(L^{1 / 5-8 / 25}\right) \underset{L \rightarrow \infty}{\longrightarrow} 0$ for large $L$, we have $t_{m}\left(k_{0}+2\right)>t_{M}\left(k_{0}\right)$ and (since $\left.V_{0} / \ell \rightarrow 0\right)$ for $\hat{t} \in\left(t_{M}\left(k_{0}\right), t_{m}\left(k_{0}+2\right)\right.$ ) the h.p. is in $(2 L / 3,(\varepsilon+2 / 3) L)$ with a velocity

$$
V \in\left(V_{m}\left(k_{0}+3\right), V_{M}\left(k_{0}-1\right)\right) \subset\left(V_{m}, V_{M}\right) \text {. }
$$

Moreover, since its collision with $(q, v)$, the h.p. has always a velocity larger in modulus than $w=e^{\lambda L}$, and therefore $\left|\hat{t}-t_{0}\right|<2 L / w=2 L e^{-\lambda L}$.

Proposition A.2. Let $v_{k}^{2}=(2 / \beta m)\left(\sqrt{k}+\log 1 / k^{2}\right), t_{k}=\exp (\sqrt{k}), k=1,2, \ldots$ and for $a>0$ consider the sets

$$
\begin{gathered}
\mathscr{R}_{k}^{\prime}(a)=\left\{x \in \mathfrak{X}_{C_{a}}: \min _{(q, v) \in S_{a, k}\left(t_{k}\right)} v>-v_{k}\right\}, \\
\mathscr{R}_{k}^{\prime \prime}(a)=\left\{x \in \mathfrak{X}_{C_{a}}: \forall t \in\left[0, t_{k}\right), \pi_{a+k}\left(t, t+\Delta_{k}\right)>M v_{k}\right\}, \\
\mathscr{R}_{k}(a)=\mathscr{R}_{k}^{\prime}(a) \cap \mathscr{R}_{k}^{\prime \prime}(a),
\end{gathered}
$$

where

$$
S_{a, b}(t)=\left\{(q, v) \in R^{2}: q \in[a, a+b)\right\} \cup C_{a+b}(0, t)
$$

and $\Delta_{k}=(a+k) / v_{k}$. Then the set

$$
\mathscr{U}_{a}^{\left(k_{0}\right)}=\bigcap_{k>k_{0}} \mathscr{R}_{k}(a)
$$

is such that $\lim _{k_{0} \rightarrow \infty} \mu^{0}\left(\mathscr{U}_{a}^{\left(k_{0}\right)}\right)=1$.

Proof. The complement $\overline{\mathscr{R}_{k}^{\prime}}=\mathfrak{X}_{C_{a}(0, \infty)} \mid \mathscr{R}_{k}^{\prime}$ has measure

$$
\begin{gathered}
\mu^{0}\left(\overline{\mathscr{R}_{k}^{\prime}}(a)\right)=1-\exp \left(-\Delta_{k}\right), \\
\mu^{0}\left(\left\{\pi_{a+k}\left(t_{1} t+\Delta_{k}\right)<M v_{k}\right\}\right) \leqq e^{-c \Delta_{k} / 4},
\end{gathered}
$$


and

$$
\mu^{0}\left(\overline{\mathscr{R}}^{\prime \prime}(a)\right) \leqq t_{k} e^{-c \Delta_{k} / 4}<e^{-c \Delta_{k} / 8},
$$

where $\overline{\mathscr{R}}_{k}^{\prime \prime}(a)=\mathfrak{X}_{C_{a}} \backslash \mathscr{R}_{k}^{\prime \prime}(a)$. The result follows from inequalities (A.12) and (A.13) by the Borel-Cantelli lemma.

Remark $A$.1. Since $\Delta_{k}$ increases with $a$, by the definition of $\mathscr{U}_{a}^{\left(k_{0}\right)}$ it follows that if $a^{\prime}>a$ and $S_{q}$ denotes the space shift, then

$$
S_{-\left(a^{\prime}-a\right)} \mathscr{U}_{a^{\prime}}^{\left(k_{0}\right)} \supset \mathscr{U}_{a}^{\left(k_{0}\right)}, \quad k_{0}=1,2, \ldots,
$$

which implies

$$
\lim _{L \rightarrow \infty} \mu^{0}\left(\mathscr{U}_{L}^{k_{0}(L)}\right)=1
$$

for any integer valued function $k_{0}(L) \uparrow \infty$.

Acknowledgements. We thank B. M. Gurevich for useful suggestions and discussions. One of the authors C.B. would like to thank the Department of Mathematics of Rutgers University for the kind hospitality. A.P. acknowledges very kind hospitality at the Moscow State University.

\section{References}

1. Sinai, Ya.G., Volkoviiski, K.L.: Funct. Anal. Appl. 5, 185-187 (1971)

2. Goldstein, S., Lebowitz, J.L., Aizenman, M.: Ergodic properties of infinite systems. In: Dynamical systems, theory, and application. Moser J., ed. Lecture Notes in Physics, Vol.38, pp. 112-143. Berlin, Heidelberg, New York: Springer 1975

3. Goldstein, S., Lebowitz, J.L., Ravishankar, K.: Ergodic properties of a system in contact with a heat bath: a one dimensional model. Commun. Math. Phys. 85, 419-427 (1982)

4. Landau, L.D., Lifschitz, E.M.: Statistical physics. New York: Pergamon 1969

5. Cornfeld, I.P., Fomin, S.V., Sinai, Ya.G.: Ergodic theory. Berlin, Heidelberg, New York: Springer 1982

Communicated by J. L. Lebowitz

Received September 5, 1984 